\title{
Adult-onset mastocytosis in the skin is highly suggestive of systemic mastocytosis
}

\author{
Sabina Berezowska ${ }^{1}$, Michael J Flaig ${ }^{2}$, Franziska Ruëff ${ }^{2}$, Christoph Walz ${ }^{1}$, \\ Torsten Haferlach ${ }^{3}$, Manuela Krokowski ${ }^{4}$, Roswitha Kerler ${ }^{1}$, Karina Petat-Dutter ${ }^{1}$, \\ Hans-Peter Horny ${ }^{3}$ and Karl Sotlar ${ }^{1}$ \\ ${ }^{1}$ Institute of Pathology, University of Munich, Munich, Germany; ${ }^{2}$ Department of Dermatology and \\ Allergology, University of Munich, Munich, Germany; ${ }^{3}$ MLL Munich Leukemia Laboratory, Munich, \\ Germany and ${ }^{4}$ Institute of Pathology, University of Schleswig Holstein, Lübeck, Germany
}

\begin{abstract}
Adult-onset urticaria pigmentosa/mastocytosis in the skin almost always persists throughout life. The prevalence of systemic mastocytosis in such patients is not precisely known. Bone marrow biopsies from 59 patients with mastocytosis in the skin and all available skin biopsies $(n=27)$ were subjected to a meticulous cytological, histological, immunohistochemical, and molecular analysis for the presence of WHO-defined diagnostic criteria for systemic mastocytosis: compact mast cell infiltrates (major criterion); atypical mast cell morphology, KIT D816V, abnormal expression of CD25 by mast cells, and serum tryptase levels $>20 \mathrm{ng} / \mathrm{ml}$ (minor criteria). Systemic mastocytosis is diagnosed when the major diagnostic criterion plus one minor criterion or at least three minor criteria are fulfilled. Systemic mastocytosis was confirmed in $\mathbf{5 7}$ patients (97\%) by the diagnosis of compact mast cell infiltrates plus at least one minor diagnostic criterion $(n=42,71 \%)$ or at least three minor diagnostic criteria $(n=15,25 \%)$. In two patients, only two minor diagnostic criteria were detectable, insufficient for the diagnosis of systemic mastocytosis. By the use of highly sensitive molecular methods, including the analysis of microdissected mast cells, KIT D816V was found in all 58 bone marrow biopsies investigated for it but only in $74 \%$ (20/27) of the skin biopsies. It is important to state that even in cases with insufficient diagnostic criteria for systemic mastocytosis, KIT D816V-positive mast cells were detected in the bone marrow. This study demonstrates, for the first time, that almost all patients with adult-onset mastocytosis in the skin, in fact, have systemic mastocytosis with cutaneous involvement.

Modern Pathology (2014) 27, 19-29; doi:10.1038/modpathol.2013.117; published online 28 June 2013
\end{abstract}

Keywords: bone marrow biopsy; cutaneous mastocytosis; KIT D816V; mastocytosis in the skin; microdissection; systemic mastocytosis; urticaria pigmentosa

Mastocytosis comprises a heterogeneous group of neoplasms characterized by an accumulation of abnormal mast cells in various tissues. The clinical course ranges from self-regressing skin lesions in children to highly malignant mast cell leukemia in adults. Two main disease variants are recognized by the WHO: cutaneous mastocytosis and systemic mastocytosis. ${ }^{1}$ Cutaneous mastocytosis typically presents as urticaria pigmentosa. Systemic mastocytosis is characterized by neoplastic mast cell infiltrates in extracutaneous tissues-almost invariably the bone marrow-and facultative skin involvement. ${ }^{1-3}$ To discriminate

Correspondence: Professor K Sotlar, MD, Institute of Pathology, University of Munich, Ludwig-Maximilians-Universität (LMU), Thalkirchner Straße 36, Munich D-80337, Germany.

E-mail: karl.sotlar@med.uni-muenchen.de

Received 25 February 2013; revised 30 April 2013; accepted 30 April 2013; published online 28 June 2013 systemic mastocytosis from isolated cutaneous mastocytosis and from other neoplastic or reactive conditions with an increased mast cell number, diagnostic criteria had been defined: The only major diagnostic criterion is the presence of multifocal compact mast cell infiltrates consisting of at least 15 mast cells in extracutaneous tissues. The four minor criteria are (1) atypical mast cell morphology ( $>25 \%$ spindle shaped), (2) aberrant mast cell immunophenotype (expression of CD25/CD2), (3) activating mutations at codon 816 of KIT, and (4) persistent elevation of baseline serum tryptase levels (>20 ng/ml). The diagnosis of systemic mastocytosis requires the fulfillment of at least the major criterion plus one minor criterion or at least three minor criteria. ${ }^{1,2}$ According to a recent consensus statement, dermatological findings suspicious for cutaneous mastocytosis should prompt a preliminary diagnosis of 'mastocytosis in the skin', with subsequent investigations being 
initiated in order to either demonstrate or rule out systemic mastocytosis. ${ }^{2}$

The aim of the present study was to precisely determine the prevalence of systemic mastocytosis in a cohort of 59 patients with adult onset mastocytosis in the skin. By addressing all WHO diagnostic criteria for systemic mastocytosis, including KIT mutational analyses in microdissected bone marrow mast cells, we were able to demonstrate that almost all patients with adult-onset mastocytosis in the skin, in fact, have systemic mastocytosis.

\section{Materials and methods}

\section{Patients}

Fifty-nine patients with the clinical diagnosis of adult-onset mastocytosis in the skin established between 2004 and 2008 at a specialized referral center at the Department of Dermatology and Allergology, Ludwig-Maximilians-Universität Munich, Munich, Germany, were investigated. Routinely, patients with suspected mastocytosis in the skin are clinically evaluated, including the occurrence of anaphylactic reactions and the determination of the type and extent of skin lesions and the baseline serum tryptase level. In addition, a skin biopsy is done in patients in whom it had not been performed by the referring dermatologist. In all the patients, bone marrow was evaluated histologically (trephine core biopsy) and cytologically (smear preparations). Additional diagnostic tests like bone density measurement or an allergological work-up were only performed when clinically indicated. The diagnosis of mastocytosis in the skin was based upon the typical maculopapular rash (major criterion for mastocytosis in the skin) and swelling upon rubbing (Darier's sign). Maculopapular lesions with marked pigmentation were diagnosed as urticaria pigmentosa and erythematous lesions without pigmentation as Teleangiectasia macularis eruptiva perstans. Determination of the extent of cutaneous disease was conducted according to Brockow et $a .^{4}$ assessing the percentage of lesional skin surface in the most intensively involved area of the whole body (A: $2 \%, B: 15 \%, C: 50 \%$, and D: $100 \%$; Figures 1a-c). Histological examinations performed in-house were assessed positive for mastocytosis in the skin when mast cell infiltrates in the skin were found with either $>15$ mast cells/cluster or $>20$ scattered mast cells per high power field $(\times 400$; minor criterion for mastocytosis in the skin). ${ }^{2}$ Retrospectively, the mast cell content in the skin was stratified semiquantitatively by anti-tryptase immunohistochemistry in in-house biopsies $(n=27 ;+,++,+++$; Figures $1 \mathrm{~d}-\mathrm{f})$. Initially, no molecular analyses for KIT D816V (minor criterion for mastocytosis in the skin) had been performed on skin biopsies.

Regarding smear preparations, mast cell cytomorphological features and numbers were assessed in toluidine blue stains outside of the crushed particles according to criteria defined by Sperr et $a l^{5}$ Routinely processed bone marrow trephine biopsy specimens were investigated by histology, immunohistochemistry, and molecular techniques applied to total DNA from complete tissue sections as described elsewhere. ${ }^{6}$ Diagnoses were established according to the published WHO criteria. ${ }^{1,2}$ The patients were stratified into three diagnostic groups: (1) systemic mastocytosis with compact mast cell infiltrates, (2) systemic mastocytosis without compact mast cell infiltrates but $\geq 3$ minor criteria, and (3) cutaneous mastocytosis without compact mast cell infiltrates and $<3$ minor criteria. Tryptase concentration was determined in blood samples obtained at least 14 days after a systemic allergic reaction by ImmunoCAP Tryptase (Phadia AB, Freiburg, Germany) according to the manufacturer's instructions. The retrospective analysis of anonymized data was approved by the local institutional review board.

\section{Tissue Processing}

Bone marrow smears were stained with toluidine blue and Pappenheim-Wright-Giemsa. All skin and bone marrow biopsy specimens were fixed in $4 \%$ neutral-buffered formalin and embedded in paraffin. Bone marrow biopsies were mildly decalcified overnight in EDTA. Tissue sections were stained with hematoxylin and eosin and Giemsa. Immunohistochemical investigations with antibodies against mast cell tryptase (1:1200; clone AA-1; Diagnostic BioSystems, Pleasanton, CA, USA), KIT/CD117 (1:400; DAKO Diagnostika, Glostrup, Denmark), and

Figure 1 Macroscopical, histological, and immunohistochemical findings. Clinical presentation of cutaneous lesions according to Brockow et al, ${ }^{4}$ assessing the percentage of lesional skin surface in the most intensively involved area of the whole body: score A ( $2 \%$, patient no. 16 ; a), score B (15\%, patient no. 35 ; b), and score C ( $50 \%$, patient no. 30 ; c). Typical anti-tryptase immunohistochemistry in skin biopsies, stratified semiquantitatively into three groups according to mast cell content. Low mast cell content (patient no. 23; d), intermediate mast cell content (patient no. 46; e), and high mast cell content (patient no. 47; f); original magnification $\times 100$. Aberrant expression of CD25 in only a subset of skin mast cells in indolent systemic mastocytosis. Moderate infiltrates $(++)$ of tryptase-positive mast cells in the upper dermis (patient no. 50; g) in a patient with KIT D816V-positive skin biopsies. Only a small subset of mast cells expresses CD25 (g). In addition, some CD25-positive cells may even be activated T-lymphocytes as suggested by the co-localization of CD3-positive lymphoid cells (h). In another case with dense mast cell infiltrates in the skin (patient no. 36; $\mathbf{i}+\mathbf{j}$ ), only few mast cells co-express CD25 as demonstrated by immunohistochemical double staining for anti-tryptase and anti-CD25 (see arrows)). Original magnification $\times 200$. 


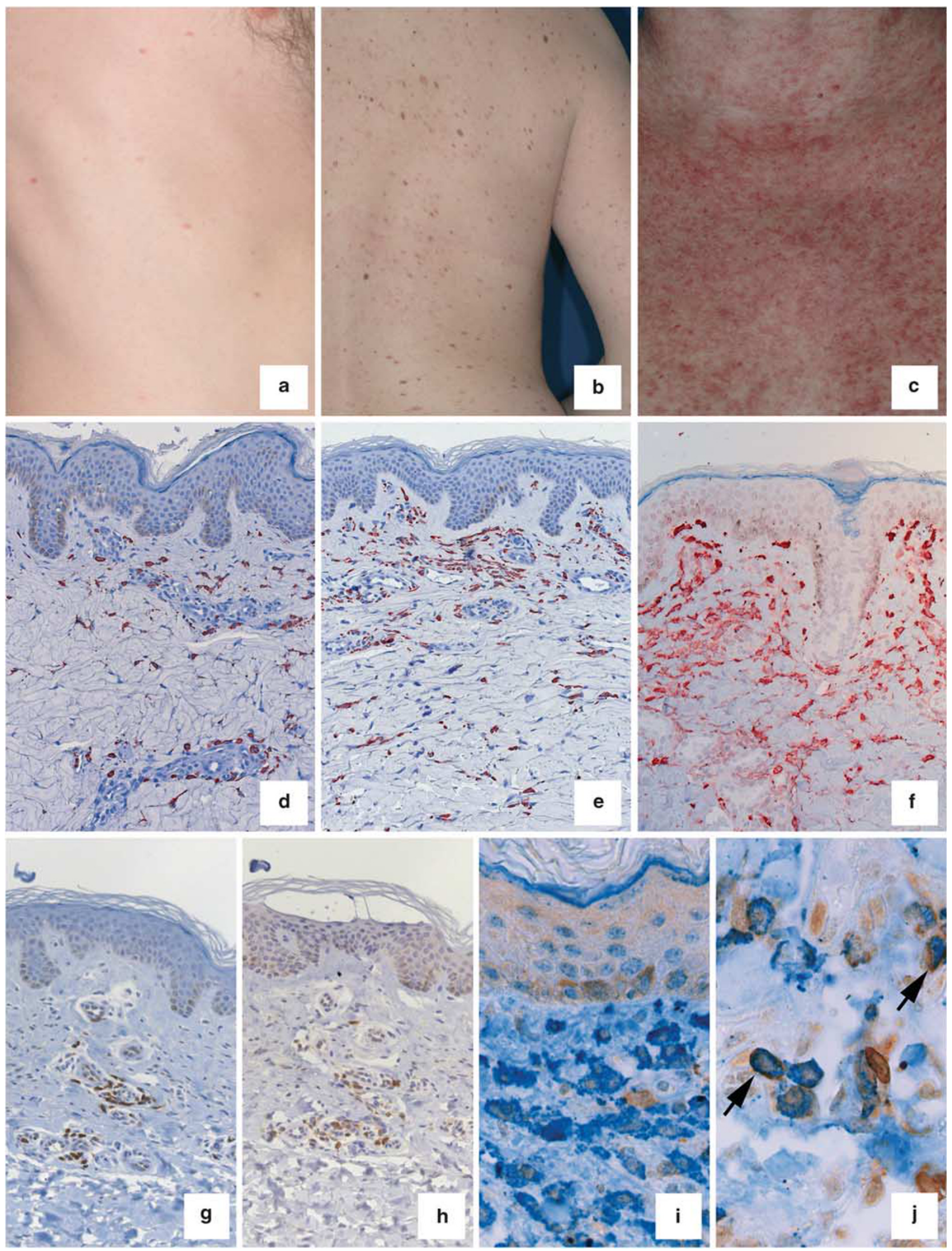


CD25 (1:50; clone 4C9; Novocastra, Newcastle, UK) were performed by the avidin-biotin immunoperoxidase (ABC) staining technique. $\mathrm{DAB}+\left(3,3^{\prime}\right.$-diaminobenzidine tetrahydrochloride, DAKO Diagnostika) served as chromogen (Figures $2 \mathrm{~b}-\mathrm{d}$ ) for diagnostic purposes, while AEC (3-amino 9 ethyl-carbazole, Zytomed, Berlin, Germany) was used for microdissection experiments.

\section{Isolation of Total DNA for Mutational Screening Analysis}

The presence of KIT codon 816 mutations was investigated in formalin-fixed and paraffin embedded skin and bone marrow biopsy specimens. Total genomic DNA was extracted from five $5-10-\mu \mathrm{m}$ paraffin sections using the QIAamp DNA Micro kit (Qiagen, Hilden, Germany) according to the manufacturer's instructions. Samples of 50-100 ng of the total extracted DNA were used for KIT mutation analyses. Genomic DNA extracted from the mast cell leukemia cell line HMC-1.2, known to heterozygously carry the KIT mutation D816V, served as the positive control. ${ }^{7}$ HMC-1.2 cells were kindly provided by Dr Butterfield, Mayo Clinic, Rochester, MN, USA.

\section{Isolation of DNA from Microdissected Mast Cells}

Single tryptase-stained cells were microdissected using the technique of laser pressure catapulting (PALM, Bernried, Germany; Figures $2 \mathrm{~g}$ and $\mathrm{h}$ ) and pooled to a total of 200 cells in the tip of a PCR tube. Microdissected cells were resuspended in $9 \mu \mathrm{l}$ of TE-buffer $\mathrm{pH}$ 8.5/0.5\% Tween ( $50 \mathrm{mM}$ Tris, $1 \mathrm{mM}$ EDTA) and incubated at $95{ }^{\circ} \mathrm{C}$ for $20 \mathrm{~min}$. After addition of proteinase $\mathrm{K}$ (Qiagen, $>600 \mu \mathrm{AU} / \mu \mathrm{l}$ ) and centrifugation, the lysate was incubated for $16-24 \mathrm{~h}$ at $56{ }^{\circ} \mathrm{C}$, followed by heat inactivation of proteinase $\mathrm{K}$. Half of the respective lysate was then amplified together with $19 \mu \mathrm{l}$ of PCR mixture with and without locked nucleic acid.

\section{DNA Amplification and Mutation Detection}

Investigations for KIT codon 816 mutations in total DNA were performed by the technique of melting point analysis of amplification products with the LightCycler System (Roche Molecular Systems, Mannheim, Germany). To increase the diagnostic sensitivity, amplicons to be genotyped were generated by nested PCR and locked nucleic acid-mediated PCR clamping. The PCR-clamping

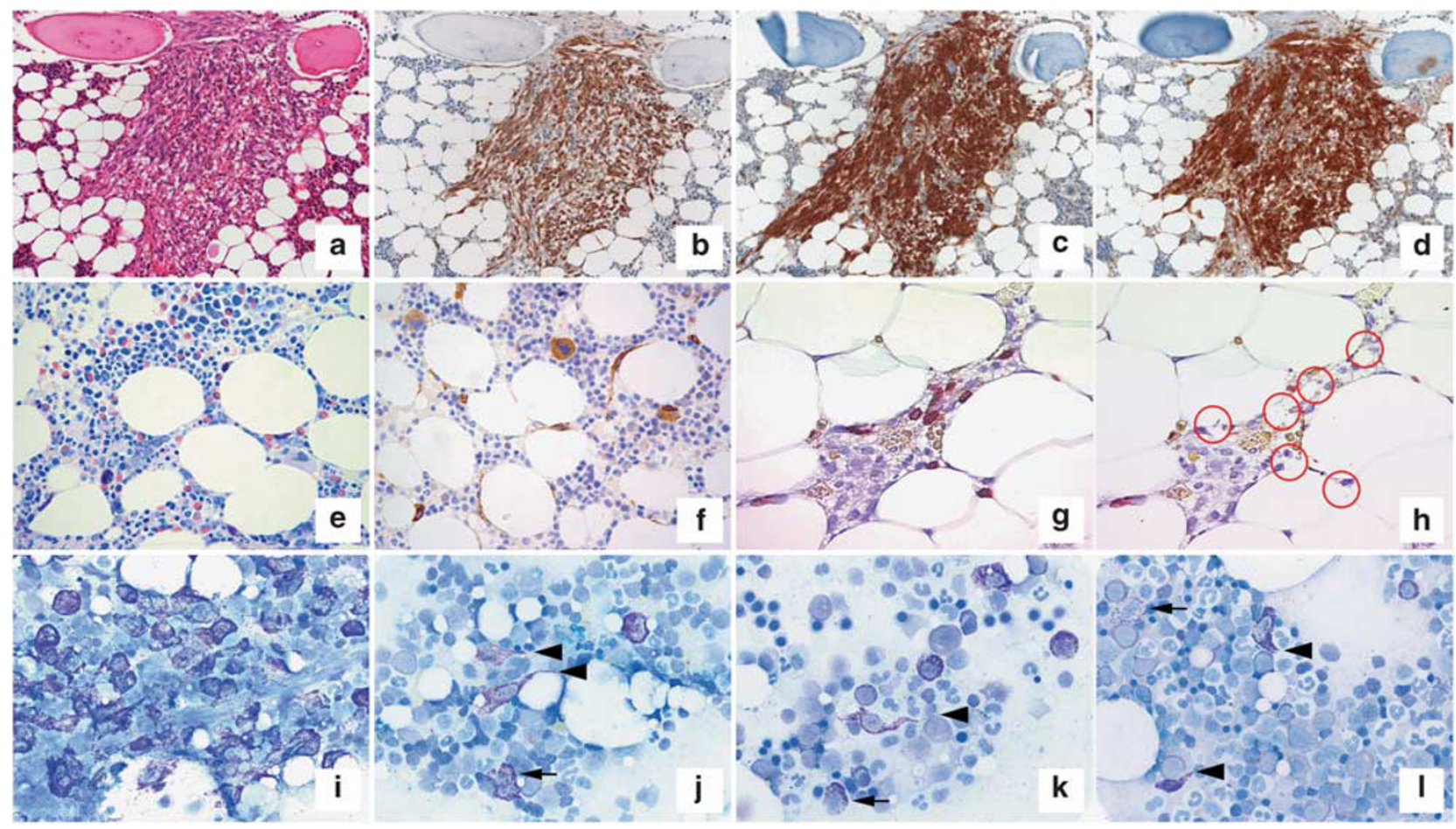

Figure 2 Typical bone marrow histology and immunochemistry in systemic mastocytosis with and without compact mast cell infiltrates. Systemic mastocytosis with compact mast cell infiltrates (patient no. 31; a-d), and systemic mastocytosis without compact mast cell infiltrates (patient no. 54; $\mathbf{e}+\mathbf{f}$ ), as depicted by hematoxylin and eosin (a), Giemsa (e), and immunohistochemical stainings for antitryptase (b), anti-CD117 (c), and anti-CD25 (d and f). Original magnification $\times 200$. Pooling of mast cells by laser pressure catapulting (LPC) in order to enhance sensitivity in detecting KIT D816V mutation. Tryptase-positive loosely scattered mast cells in bone marrow ( $\mathbf{g}$ and $\mathbf{h}$ ), before LPC (g) and after LPC (h). Original magnification $\times 400$. In bone marrow aspirate smears, increased amounts of mast cells are easily appreciated close to the crushed particles (i). Abnormal mast cell morphology included spindle-shaped appearence and cytoplasmic projections (arrowheads; $\mathbf{j}-\mathbf{l}$ ) as well as enlarged oval eccentric nuclei and hypogranulation of the cytoplasm (arrows; $\mathbf{j}-\mathbf{l}$ ). Original magnification $\times 63$ 
step was only performed during the first round amplification.

PCRs were performed in reaction volumes of $25 \mu \mathrm{l}$ containing final concentrations of $1 \times$ buffer, $10 \mathrm{mM}$ Tris-HCl pH 8.3, $50 \mathrm{mM} \quad \mathrm{KCl}, \quad 1.5 \mathrm{mM} \quad \mathrm{MgCl}_{2}$ (Applied Biosystems, Darmstadt, Germany), $200 \mu \mathrm{M}$ dNTPs each (Fermentas, St. Leon-Rot, Germany), $30 \mathrm{mU} / \mu \mathrm{l}$ thermostable DNA polymerase (AmpliTaq Gold DNA polymerase; Roche Molecular Systems), $0.3 \mu \mathrm{M}$ of each primer, and $25 \mathrm{nM}$ locked nucleic acid, using first round primers c-kit $1 \mathrm{~F} / 1 \mathrm{~B}$ and second round primers c-kit $2 \mathrm{~F} / 2 \mathrm{~B}$ (Table 1). ${ }^{6}$ All primers and probes and the locked nucleic acid molecule were obtained from TIB Molbiol (Berlin, Germany). The reaction conditions for both amplification rounds were as follows: $95^{\circ} \mathrm{C}^{10: 00}$ $\left(95^{\circ} \mathrm{C}^{0: 30} ; 56^{\circ} \mathrm{C}^{0: 30} ; 72^{\circ} \mathrm{C}^{0: 30}\right)_{40} ; 72^{\circ} \mathrm{C}^{5: 00} ; 10^{\circ} \mathrm{C}^{10: 00}$. Detection of KIT D816V in microdissected mast cells required slight modifications for the first PCR amplification round, which was performed as a time-release PCR: $95{ }^{\circ} \mathrm{C}^{0: 45}\left(95^{\circ} \mathrm{C}^{0: 30} ; \quad 56{ }^{\circ} \mathrm{C}^{0: 30}\right.$; $\left.72{ }^{\circ} \mathrm{C}^{0: 30}\right)_{55} ; 72{ }^{\circ} \mathrm{C}^{5: 00} ; 10{ }^{\circ} \mathrm{C}^{10: 00}$. One microliter of the PCR products was used as template for nested PCR amplifications. All PCRs were run on a Veriti 96-well thermal cycler (Applied Biosystems, Weiterstadt, Germany). For the genotyping of nested PCR products, $10 \mu \mathrm{l}$ of the amplification products were added to $10 \mu \mathrm{l}$ of hybridization mixture, resulting in a final concentration of $0.3 \mu \mathrm{M}$ of hybridization probes ckit-sensor/-anchor (TIB Molbiol; Table 1) and $2.78 \mathrm{mM} \mathrm{MgCl}_{2}$. Subsequent melting point analysis was performed on the Light Cycler System (Roche Molecular Systems). ${ }^{8}$ After denaturing of samples for $5 \mathrm{~min}$ at $95^{\circ} \mathrm{C}$, melting curve analysis started at $40{ }^{\circ} \mathrm{C}$, the temperature being increased by $0.3^{\circ} \mathrm{C} / \mathrm{s}$ to $95^{\circ} \mathrm{C} .{ }^{7}$

\section{Statistical Analysis}

The Fisher's exact test for $2 \times 2$ contingency tables was used to test for differences in proportions of nominal variables. The Kruskal-Wallis test or Mann-Whitney $U$ test were applied to test for differences in medians of continuous variables. Correlation between continuous variables was determined with the nonparametric Spearman correlation coefficient. The significance level was set at 0.05. Statistical analysis of the data was carried out using the IBM SPSS software, version 18.0 (SPSS, Chicago, IL).

\section{Results}

\section{Clinical and Laboratory Findings}

Details of the patient characteristics are listed in Table 2, and their analysis are summarized in Table 3. The median age at onset of the cutaneous symptoms was 35 years (range 15-63 years) and the median duration until presentation was 9.5 years (range 0-37 years). It could not be determined exactly in 13 patients. None of the patients had morphological or clinical features of smouldering systemic mastocytosis, aggressive systemic mastocytosis, or mast cell leukemia. In particular, the so-called ' $\mathrm{B}$ ' findings and ' $\mathrm{C}$ ' findings were missing. ${ }^{1}$ The severity of the skin lesions (A-D; Figures 1a-c) did not correlate with age, sex, tryptase levels, or the three diagnostic groups (systemic mastocytosis with compact mast cell infiltrates, systemic mastocytosis without compact mast cell infiltrates, and cutaneous mastocytosis). Between patients with and without compact mast cell infiltrates, no statistically significant differences in age at onset of cutaneous symptoms, duration, age at the time of investigation, and sex was found. Anaphylactic reactions, most often caused by Hymenoptera stings $(n=19)$, were significantly more frequent among indolent systemic mastocytosis patients without detectable compact mast cell infiltrates (12/14 (86\%) vs 15/41 (37\%), $P=0.002)$. Pathological bone density measurements were observed in $60 \%$ of the evaluated patients $(31 / 52)$. Reduced bone density was associated with elevated tryptase levels $(P=0.01$ for continuous tryptase levels, $r=-0.353)$. Serum tryptase was elevated

Table 1 Sequences of PCR primers and hybridization probes

\begin{tabular}{llc}
\hline Name & Sequence $\left(5^{\prime}-3^{\prime}\right)$ & Positions (nt) $^{\text {GB no. }}{ }^{\text {a }}$ \\
\hline $\begin{array}{l}\text { Primers } \\
\text { c-kit 1F }\end{array}$ & CAC AGA GAC TTG GCA GCC AG & $7097-7116$ \\
C-kit 1B & CAG GAT TTA CAT TAT GAA AGT CAC GG & $7309-7284$ \\
c-kit 2F & CAG CCA GAA ATA TCC TCC TTA CT & $7110-7132$ \\
c-kit 2B & TTG CAG GAC TGT CAA GCA GAG & $7247-7227$ \\
Probes & & $7168-7193$ \\
Sensor & AGC CAG AGT CAT CAA GAA TGA TTC TA (Fl) & $7197-7214$ \\
Anchor & (LC) ATG TGG TTA AAG GAA ACG TGA GTA CCC A (p) ${ }^{\mathrm{c}}$ & $7169-7187$ \\
LNA & GCC AGA GAC ATC AAG AAT G & \\
\hline
\end{tabular}

${ }^{\mathrm{a}} \mathrm{GB}$ no., GenBank accession number.

${ }^{\mathrm{b}}$ The sensor probes were $3^{\prime}$-fluorescein labeled (Fl).

${ }^{\mathrm{c}}$ The anchor probes were $5^{\prime}$-terminal labeled with LightCycler Red 640 (LC) and 3'-terminal blocked with a phosphate group (p). 
Table 2 Patient characteristics

\begin{tabular}{|c|c|c|c|c|c|c|c|c|c|c|c|c|c|c|c|c|c|c|}
\hline \multirow[b]{2}{*}{$\begin{array}{l}\text { Pt } \\
\text { no. }\end{array}$} & \multirow[b]{2}{*}{$\begin{array}{l}\text { Age } \\
x / y\end{array}$} & \multirow[b]{2}{*}{ Sex } & \multirow[b]{2}{*}{ Diagnosis } & \multicolumn{5}{|c|}{ Bone marrow findings } & \multirow[b]{2}{*}{$\begin{array}{c}\text { Serum } \\
\text { tryptase } \\
(\mu \mathrm{g} / \mathrm{l})\end{array}$} & \multicolumn{5}{|c|}{ Skin findings } & \multicolumn{3}{|c|}{ Clinical findings } & \\
\hline & & & & $\begin{array}{l}M C^{\mathrm{a}} \\
(\%)\end{array}$ & $C M C I$ & $S P$ & $C D 25$ & KIT & & $\begin{array}{c}\text { Brockow- } \\
\text { score } \\
\text { skin }\end{array}$ & $\begin{array}{c}M C \\
\text { density }\end{array}$ & $\begin{array}{l}\text { MC-infiltration } \\
\text { pattern }\end{array}$ & $\begin{array}{c}C D 25+M C \\
(\%)\end{array}$ & KIT & $\begin{array}{l}\text { Reason for } \\
\text { presentation }\end{array}$ & Anaphylaxis & $\begin{array}{c}\text { Bone } \\
\text { density } \\
(\Delta T)\end{array}$ & \\
\hline 1 & $32 / 38$ & $\mathrm{f}$ & ISM & $<1$ & + & + & + & D816V ( $\mu$ ) & 10,3 & A & ND & ND & ND & ND & UP & 4 & & \\
\hline 2 & $24 / 30$ & $\mathrm{~m}$ & ISM & $1-3$ & + & + & + & D816V & 159 & $\mathrm{C}$ & ++ & 1 & 10,0 & wt & Fractures & - & $-2,1$ & \\
\hline 3 & $\mathrm{ND} / 54$ & $\mathrm{f}$ & ISM & 1 & + & + & + & D816V & 42 & A & ND & ND & ND & ND & UP & 3 & $-1,3$ & \\
\hline 4 & $41 / 53$ & $\mathrm{f}$ & ISM & 5 & + & + & + & D816V & 26 & A & ND & ND & ND & ND & UP & 2,5 & $-2,6$ & \\
\hline 5 & $36 / 40$ & $\mathrm{~m}$ & ISM & 1 & + & + & + & D816V & 14,4 & A & + & 1 & 25 & D816V & UP & - & $-0,9$ & \\
\hline 6 & $\mathrm{ND} / 56$ & $\mathrm{f}$ & ISM & 15 & + & + & + & D816V & 37,2 & A & ++ & 1 & 25 & wt & HVA & 2 & $-0,3$ & \\
\hline 7 & $31 / 42$ & $\mathrm{f}$ & ISM & $1-3$ & + & + & + & D816V & 58 & A & ND & ND & ND & ND & UP & - & $-1,3$ & \\
\hline 8 & $60 / 65$ & $\mathrm{f}$ & ISM & $1-3$ & + & + & + & D816V & 40,2 & A & +++ & 1 & 7,5 & D816V ( $\mu)$ & UP & - & $-1,3$ & \\
\hline 9 & $39 / 43$ & $\mathrm{~m}$ & ISM & 1 & + & + & + & D816V & 22,9 & A & ND & ND & ND & ND & UP & - & $-0,3$ & \\
\hline 10 & $25 / 57$ & $\mathrm{~m}$ & ISM & 1 & + & + & + & D816V & 10,9 & A & +++ & 1 & 25 & D816V & UP & - & 0,09 & \\
\hline 11 & $17 / 32$ & $\mathrm{f}$ & ISM & 5 & + & + & + & D816V & 49 & $\mathrm{C}$ & ND & ND & ND & ND & UP & 4 & $-1,7$ & \\
\hline 12 & $63 / 71$ & $\mathrm{f}$ & ISM & 5 & + & + & + & D816V & 36,9 & $\mathrm{C}$ & ND & ND & ND & ND & UP & - & $-3,5$ & \\
\hline 13 & $\mathrm{ND} / 46$ & $\mathrm{~m}$ & ISM & 1 & + & + & + & D816V & 26,3 & A & + & 1 & 35 & wt & HVA & 2 & $-2,4$ & \\
\hline 14 & $40 / 68$ & $\mathrm{~m}$ & ISM & $3-5$ & + & + & + & D816V & 87 & $\mathrm{~B}$ & ND & ND & ND & ND & UP & 1 & $-2,99$ & n \\
\hline 15 & $36 / 38$ & $\mathrm{~m}$ & ISM & 1 & + & + & + & D816V & 12,9 & A & ND & ND & ND & ND & UP & - & $-0,2$ & 罣 \\
\hline 16 & $\mathrm{ND} / 35$ & $\mathrm{~m}$ & ISM & $<5$ & + & + & + & D816V & 51 & A & ND & ND & ND & ND & HVA & 2 & $-2,1$ & $\frac{D}{0}$ \\
\hline 17 & $24 / 26$ & $\mathrm{f}$ & ISM & 1 & + & + & + & D816V & 20,6 & A & ND & ND & ND & ND & UP & - & $-1,7$ & 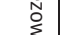 \\
\hline 18 & $21 / 22$ & $\mathrm{f}$ & ISM & 5 & + & + & + & D816V & 13,6 & A & ND & ND & ND & ND & UP & - & & $\sum_{n=1}^{n}$ \\
\hline 19 & $15 / 28$ & $\mathrm{f}$ & ISM & $1-2$ & + & + & + & D816V & 12,4 & $\mathrm{~B}$ & ++ & 1 & 2 & D816V $(\mu)$ & UP & - & 0,3 & $\hat{\tilde{N}}$ \\
\hline 20 & $38 / 42$ & $\mathrm{f}$ & ISM & 3 & + & + & + & D816V & 15 & $\mathrm{C}$ & ND & ND & ND & ND & HVA & 2,3 & 0,3 & $\frac{9}{0}$ \\
\hline 21 & $25 / 31$ & $\mathrm{~m}$ & ISM & 1 & + & + & + & D816V & 22,7 & $\mathrm{~B}$ & ++ & 1 & 15 & D816V & UP & - & $-0,9$ & \\
\hline 22 & $20 / 26$ & $\mathrm{f}$ & ISM & 3 & + & + & + & D816V & 89 & A & ND & ND & ND & ND & UP & NA & & \\
\hline 23 & $\mathrm{ND} / 51$ & $\mathrm{f}$ & ISM & 10 & + & + & + & D816V & 32,4 & $\mathrm{~B}$ & + & 3 & 45 & D816V & HVA & 2 & $-2,8$ & \\
\hline 24 & $\mathrm{ND} / 66$ & $\mathrm{~m}$ & ISM & $1-2$ & + & + & + & D816V & 25,8 & A & ++ & 1 & 7,5 & wt & HVA & 2 & $-0,6$ & \\
\hline 25 & $23 / 33$ & $\mathrm{~m}$ & ISM & $30-35$ & + & + & + & D816V & 156,5 & C & ND & ND & ND & ND & UP & - & 0,4 & \\
\hline 26 & $\mathrm{ND} / 32$ & $\mathrm{f}$ & ISM & 1 & + & + & + & D816V & 12,4 & A & ND & ND & ND & ND & HVA & 2 & & \\
\hline 27 & $45 / 51$ & $\mathrm{~m}$ & ISM & $3-5$ & + & + & + & D816V & $\begin{array}{l}12,7 \\
39,6\end{array}$ & A & ND & ND & ND & ND & UP & - & $-1,4$ & \\
\hline 28 & $35 / 42$ & $\mathrm{~m}$ & ISM & 1 & + & + & + & D816V & 7,5 & A & ND & ND & ND & ND & HVA & 2 & & \\
\hline 29 & $51 / 61$ & $\mathrm{~m}$ & ISM & 3 & + & + & + & D816V & 77,6 & $\mathrm{~B}$ & ND & ND & ND & ND & UP & - & $-3,2$ & \\
\hline 30 & $42 / 45$ & $\mathrm{f}$ & ISM & $20-25$ & + & + & + & D816V & 106,1 & $\mathrm{C}$ & ND & ND & ND & ND & UP & - & $-0,4$ & \\
\hline 31 & $57 / 60$ & $\mathrm{f}$ & ISM & 10 & + & + & + & D816V & 66,1 & $\mathrm{C}$ & ND & ND & ND & ND & UP & - & $-1,4$ & \\
\hline 32 & $\mathrm{ND} / 54$ & $\mathrm{~m}$ & ISM & $1-2$ & + & + & + & D816V & 32 & $\mathrm{~B}$ & + & 1 & 75 & wt & RA & - & 0,25 & \\
\hline 33 & $42 / 45$ & $\mathrm{~m}$ & ISM & 1 & + & - & + & $\mathrm{D} 816 \mathrm{~V}(\mu)$ & 16,4 & A & ND & ND & ND & ND & UP & - & $-0,3$ & \\
\hline 34 & $25 / 46$ & $\mathrm{~m}$ & ISM & $20-25$ & + & + & + & D816V & 280,5 & $\mathrm{~B}$ & +++ & 3 & 15 & D816V ( $\mu)$ & UP & - & $-1,2$ & \\
\hline 35 & $19 / 30$ & $\mathrm{f}$ & ISM & 1 & + & + & + & $\mathrm{NA}^{\mathrm{b}}$ & 8,4 & $\mathrm{~B}$ & +++ & 2 & 7,5 & wt & UP & - & $-1,6$ & \\
\hline 36 & $35 / 72$ & $\mathrm{~m}$ & ISM & $<5$ & + & + & + & D816V & 138,5 & $\mathrm{C}$ & +++ & 2 & 2 & D816V & UP & - & $-1,8$ & \\
\hline 37 & $36 / 45$ & $\mathrm{~m}$ & ISM & 1 & + & + & + & $\mathrm{D} 816 \mathrm{~V}(\mu)$ & 41,5 & $\mathrm{C}$ & ND & ND & ND & ND & UP & - & $\begin{array}{l}1,0 \\
-2,3\end{array}$ & \\
\hline 38 & $\mathrm{ND} / 35$ & $\mathrm{~m}$ & ISM & 1 & + & + & + & D816V & 17,4 & A & ++ & 1 & 7,5 & D816V & HVA & 2 & & \\
\hline 39 & $40 / 54$ & $\mathrm{~m}$ & ISM & $1-2$ & + & + & + & D816V & 25,9 & $\mathrm{C}$ & ND & ND & ND & ND & HVA & 2 & $-1,62$ & \\
\hline 40 & $23 / 44$ & $\mathrm{f}$ & ISM & $5-10$ & + & + & + & D816V & 146,5 & $\mathrm{C}$ & ND & ND & ND & ND & UP & - & $\begin{array}{l}-1,7 \\
-1,7\end{array}$ & \\
\hline 41 & $18 / 34$ & $\mathrm{f}$ & ISM & $5-10$ & + & + & + & D816V & 28 & $\mathrm{~B}$ & ND & ND & ND & ND & UP & - & 0,2 & \\
\hline 42 & $41 / 45$ & $\mathrm{~m}$ & ISM & 3 & + & + & + & D816V & 32,7 & A & ND & ND & ND & ND & UP & - & 0,5 & \\
\hline 43 & $36 / 36$ & $\mathrm{~m}$ & ISM & $<1$ & - & + & + & D816V & 8,4 & A & ND & ND & ND & ND & UP & 5 & $-2,3$ & \\
\hline 44 & $29 / 54$ & $\mathrm{f}$ & ISM & $<1$ & - & - & + & D816V ( $\mu$ ) & 33,3 & A & ND & ND & ND & ND & HVA & 2 & $-1,3$ & \\
\hline 45 & $\mathrm{ND} / 30$ & $\mathrm{f}$ & ISM & $<1$ & - & + & + & D816V & 16 & $\mathrm{~B}$ & ++ & 1 & 15 & D816V & UP & NA & $-1,9$ & \\
\hline 46 & $21 / 22$ & $\mathrm{~m}$ & ISM & 1 & - & + & + & D816V & 28,5 & A & ++ & 1 & 25 & D816V & UP & - & $-1,8$ & \\
\hline 47 & $\mathrm{ND} / 39$ & $\mathrm{~m}$ & ISM & $<1$ & - & + & + & D816V ( $\mu$ ) & 9,9 & A & +++ & 2 & 35 & D816V ( $\mu)$ & HVA & 2 & $-0,4$ & \\
\hline 48 & $\mathrm{ND} / 42$ & $\mathrm{f}$ & ISM & 1 & - & + & + & D816V & 10,4 & A & +++ & 1 & 25 & wt & HVA & 2 & & \\
\hline 49 & $\mathrm{ND} / 40$ & $\mathrm{~m}$ & ISM & $<1$ & - & + & - & D816V ( $\mu$ ) & 20,8 & A & +++ & 1 & 7,5 & D816V & HVA & 2 & 0,86 & \\
\hline 50 & $15 / 27$ & $\mathrm{f}$ & ISM & 1 & - & + & + & D816V & $\begin{array}{l}20,0 \\
9,8\end{array}$ & $\mathrm{~B}$ & $\begin{array}{l}+1 \\
++\end{array}$ & 1 & 7,5 & D816V & NCN & - & 1,4 & \\
\hline 51 & $17 / 43$ & $\mathrm{~m}$ & ISM & 1 & - & + & + & D816V & 16,5 & A & + & 3 & 45 & D816V & HVA & 2 & $\begin{array}{l}1,7 \\
-2,6\end{array}$ & \\
\hline 52 & $26 / 46$ & $\mathrm{f}$ & ISM & $<1$ & - & + & + & D816V & 25,3 & A & ++ & 1 & 7,5 & D816V & UP & 3 & $-1,4$ & \\
\hline
\end{tabular}


$>20 \mathrm{ng} / \mathrm{ml}$ in $36 / 59(61 \%)$ patients, $30(83 \%)$ of whom had compact mast cell infiltrates in the bone marrow. Serum tryptase levels correlated significantly with bone marrow involvement as defined for the three diagnostic groups: systemic mastocytosis with compact mast cell infiltrates, systemic mastocytosis without compact mast cell infiltrates, and cutaneous mastocytosis $(P=0.003$ for continuous tryptase levels and $P=0.004$ for grouped tryptase levels).

\section{Bone Marrow Morphology and Molecular Pathology}

Cytological, histological, and immunohistochemical evaluation of the bone marrow revealed compact mast cell infiltrates in $71 \%(42 / 59)$ of the patients, expression of CD25 in 95\% (56/59), and abnormal mast cell morphology of $>25 \%$ of mast cells in $95 \%$ (56/59). Abnormal mast cell morphology included spindle-shaped appearance, cytoplasmic projections, enlarged oval eccentric nuclei, and hypogranulation of the cytoplasm. Most of these cells corresponded to the so-called 'atypical mast cells Type I' ${ }^{5}$ The area of mast cell infiltrates in the bone marrow showed a significant association with the three diagnostic groups (systemic mastocytosis with compact mast cell infiltrates, systemic mastocytosis without compact mast cell infiltrates, and cutaneous mastocytosis; $P<0.001$ ). The KIT D816V mutation was detected in genomic DNA from paraffin sections in $48(83 \%)$ of the 58 patients in whom it was performed. Seventy-nine percent (38/48) of these patients had compact mast cell infiltrates (Figures 2a-d). Interestingly, further analysis of microdissected mast cells revealed KIT D816V in all of the 10 hitherto apparently KIT wild-type cases. Among them only 3 patients $(3 \%)$ had detectable compact mast cell infiltrates. Thus, the necessity to enrich mast cells by microdissection in order to detect KIT D816V correlated significantly with the bone marrow mast cell burden as reflected by the presence or absence of compact mast cell infiltrates $(P=0.004)$ and the area of mast cells among nucleated bone marrow cells $(<1 \%$ : $10 / 31$, and $>1 \%$ : $0 / 27 ; P=0.015)$. Altogether, $97 \%(57 / 59)$ of the patients fulfilled the WHO criteria for the diagnosis of systemic mastocytosis. In 15 patients $(25 \%)$, the diagnosis of systemic mastocytosis was based upon the presence of $3(n=12)$ or even 4 $(n=3)$ minor diagnostic criteria in the absence of compact mast cell infiltrates (Figures 2e and f). These patients had a very low mast cell burden of $<1 \%$ of the bone marrow area and diagnostically elevated serum tryptase levels in only $40 \%$ (compared with $83 \%$ of patients with compact mast cell infiltrates). Two (3\%) patients fulfilled only one or two minor criteria (patient no. 58 and 59, Table 2), insufficient for the diagnosis of systemic mastocytosis, even though clonality was demonstrated by KIT D816V-positivity in mast cells microdissected from bone marrow sections. 
Table 3 Correlation of clinical, morphological, and molecular results

\begin{tabular}{|c|c|c|c|c|c|c|c|}
\hline & $\begin{array}{c}\text { No. }(\%) \\
\text { of patients }\end{array}$ & $\begin{array}{l}\text { Median } \\
\text { (range) }\end{array}$ & $\begin{array}{l}\text { SM with } \\
\text { CMCI }\end{array}$ & $\begin{array}{c}S M, \geq 3 \\
\text { minor } \\
\text { criteria }\end{array}$ & $\begin{array}{c}\text { CM, } \\
<3 \text { minor } \\
\text { criteria }\end{array}$ & $\begin{array}{c}\text { P value of } \\
\text { SM with } \\
\text { CMCI vs } S M \\
\text { without DMCI }\end{array}$ & $\begin{array}{l}\text { Overall } \\
\mathrm{P} \text { value }\end{array}$ \\
\hline Total no. of patients & 59 & & 42 & 15 & 2 & & \\
\hline Male & $31 / 59(53)$ & & 22 & 8 & 1 & NS $(1.0)^{*}$ & NS $(1.0)^{*}$ \\
\hline Female & $28 / 59(47)$ & & 20 & 7 & 1 & & \\
\hline Age (years) & & $43(22-72)$ & $44(22-72)$ & $40(22-65)$ & $46.5(44-49)$ & NS $(0.346)^{\$}$ & NS $(0.541)^{\S}$ \\
\hline $\begin{array}{l}\text { Age at first presentation of } \\
\text { cutaneous symptoms }(N=46)\end{array}$ & & $35(15-63)$ & $35(15-63)(n=33)$ & $29(15-42)(n=11)$ & $38(37-39)(n=2)$ & NS $(0.179)^{\$}$ & NS $(0.257)^{\S}$ \\
\hline Anaphylaxis $(n=57)$ & $27 / 57(47)$ & & $15(n=41)$ & $12(n=14)$ & 0 & $0.002 *$ & 0.001 * \\
\hline \multicolumn{8}{|l|}{ Bone involvement $(\mathrm{n}=52)$} \\
\hline Osteoporosis with fracture & $2 / 52(4)$ & & 2 & 0 & 0 & NS $(0.479)^{*}$ & NS $(0.715)^{*}$ \\
\hline Osteoporosis & $7 / 52(14)$ & & 4 & 3 & 0 & & \\
\hline Osteopenia & $21 / 52(40)$ & & 15 & 5 & 1 & & \\
\hline Osteosklerosis & $1 / 52(2)$ & & 0 & 1 & 0 & & \\
\hline Normal bone density & $21 / 52(40)$ & & 15 & 5 & 1 & & \\
\hline \multicolumn{8}{|l|}{ Dermatological manifestation } \\
\hline A & $35 / 59(59)$ & & 22 & 11 & 2 & NS $(0.273)^{*}$ & NS $(0.448)^{*}$ \\
\hline $\mathrm{B}$ & $12 / 59(20)$ & & 9 & 3 & 0 & & \\
\hline $\mathrm{C}$ & $12 / 58(20)$ & & 11 & 1 & 0 & & \\
\hline $\mathrm{D}$ & 0 & & 0 & 0 & 0 & & \\
\hline \multicolumn{8}{|l|}{ Serum-Tryptase $(\mu \mathrm{g} / \mathrm{l})$} \\
\hline$<11,4$ & $12 / 59(20)$ & & 4 & 7 & 1 & $0.009 *$ & 0.004 * \\
\hline $11,4-20$ & $11 / 59(19)$ & & 8 & 2 & 1 & & \\
\hline$>20$ & $36 / 59(61)$ & & 30 & 6 & 0 & & \\
\hline Median (range) & & $25.8(5.6-280.5)$ & $32.3(7.5-280.5)$ & $16(5.6-48.6)$ & $10.2(5.8-14.6)$ & $0.002^{\$}$ & $\mathbf{0 . 0 0 3}^{\S}$ \\
\hline \multicolumn{8}{|l|}{ Bone Marrow findings } \\
\hline \multicolumn{8}{|l|}{ Area MC/nucleated BM cells } \\
\hline$\leq 1 \%$ & $32 / 59(54)$ & & 15 & 15 & 2 & $<0.001 *$ & $<0.001 *$ \\
\hline$>1-5 \%$ & $19 / 59(32)$ & & 19 & 0 & 0 & & \\
\hline$>5-20 \%$ & $5 / 59(9)$ & & 5 & 0 & 0 & & \\
\hline$>20 \%$ & $3 / 59(5)$ & & 3 & 0 & 0 & & \\
\hline \multicolumn{8}{|l|}{ MC morphology } \\
\hline Spindled shape & $56 / 59(95)$ & & 41 & 14 & 1 & NS $(0.662)^{*}$ & NS \\
\hline Round shape & $3 / 59(5)$ & & 1 & 1 & 1 & & \\
\hline \multicolumn{8}{|l|}{ CD25 immunophenotype } \\
\hline Positive & $56 / 59(95)$ & & 42 & 14 & 0 & NS $(0.461)^{*}$ & $<0.001^{*}$ \\
\hline Negative & $3 / 59(5)$ & & 0 & 1 & 2 & & \\
\hline \multicolumn{8}{|l|}{$c$-kit $(\mathrm{n}=58)$} \\
\hline D816V—total DNA & 48/58 (83) & & 38 & 10 & 0 & 0.026 * & $0.001 *$ \\
\hline D816V_pooled MC & $10 / 10(100)$ & & 3 & 5 & 2 & & \\
\hline \multicolumn{8}{|l|}{ Skin biopsy findings $(\mathrm{n}=27)$} \\
\hline \multicolumn{8}{|l|}{ MC density } \\
\hline+ & $5 / 27(19)$ & & 4 & 1 & 0 & NS $(0.754)^{*}$ & NS $(0.915)^{*}$ \\
\hline++ & $12 / 27(44)$ & & 6 & 5 & 1 & & \\
\hline+++ & $10 / 27(37)$ & & 5 & 4 & 1 & & \\
\hline \multicolumn{8}{|l|}{$C D 25$} \\
\hline CD25-positive MC/total MC (\%) & & $15(2-75)$ & $15(2-75)$ & $20(7.5-45)$ & 0 & NS $(0.843)^{\$}$ & NS $(0.273)^{\S}$ \\
\hline \multicolumn{8}{|l|}{$c$-kit } \\
\hline D816V—-total DNA & $14 / 27(52)$ & & 6 & 8 & 0 & NS $(0.181)^{*}$ & $0.039 *$ \\
\hline D816V_pooled MC & $6 / 27(22)$ & & 3 & 1 & 2 & & \\
\hline wt & $7 / 27(26)$ & & 6 & 1 & 0 & & \\
\hline
\end{tabular}

MC, mast cells; CMCI, compact MC infiltrates; SM, systemic mastocytosis; CM, cutaneous mastocytosis; UP, urticaria pigmentosa.

Brockow-score skin: percentage of lesional skin surface in the most intensively involved area of the whole body. A, $<2 \%$;,$<15 \%$; C, $<50 \%$; D, 50-100\%. NS, non significant; values in bold depict results which meet statistical significance; wt, wild type; ${ }^{*}$ Fisher's exact test; \$Mann-Whitney-U Test; §Kruskal-Wallis test.

Morphological signs of an associated hematological non-mast cell lineage disease were not detected in any of our patients.

\section{Skin Histology and Molecular Pathology}

In addition to the typical skin rash, a diagnostic increase in mast cells was observed in all the available skin biopsies $(n=27)$, leading to the preliminary diagnosis of mastocytosis in the skin (Figures $2 \mathrm{~d}$ and e). Mast cell density in the skin did not correlate with the macroscopic intensity of the skin $\operatorname{rash}(P=0.912)$.

Analysis of DNA from the paraffin sections revealed KIT D816V in 52\% (14/27) of the patients. Microdissected mast cells harbored KIT D816V in 
additional $6(22 \%)$ cases, including both patients with cutaneous mastocytosis. In the remaining 7 cases, KIT D816V could not be detected even when microdissected skin mast cells were analyzed, despite the fact that all patients harbored this mutation in their bone marrow mast cells. Interestingly, $86 \%(6 / 7)$ of these patients had systemic mastocytosis with detectable compact mast cell infiltrates in contrast to only $45 \%(9 / 20)$ in whom KIT D816V was detected in the skin. The molecular finding of KIT wild-type in the skin did neither correlate with the macroscopic intensity of the skin rash nor with the mast cell density in the skin or in the bone marrow $(P=1.0, P=0.56, P=0.09)$. Furthermore, it was not associated with the percentage of CD25-expressing mast cells in the skin $(P=0.693$, Figures $1 \mathrm{~g}-\mathrm{j}$ ).

\section{Discussion}

In the present study, systematic investigation of the bone marrow, including histology, immunohistochemistry, and molecular analysis for KIT D816V as well as determination of the serum tryptase levels led to a diagnosis of systemic mastocytosis in $97 \%$ $(57 / 59)$ of patients with adult-onset mastocytosis in the skin and detection of clonal KIT D816V-positive mast cells in the remaining two cases. In previous studies investigating patients with mastocytosis in the skin, the frequency of systemic disease ranged from 41 to $73 \% .^{9-12}$

Bone marrow is the tissue of choice for KIT mutational analysis. It was found clearly preferable over skin in the present study in which KIT D816V was detected in all the bone marrow specimens investigated (58/58) but in only $74 \%(20 / 27)$ of lesional skin biopsies. The latter finding is consistent with the 77\% (86/112) prevalence of KIT D816V in skin biopsies found by Lanternier et al. ${ }^{9}$ However, underlying systemic disease was found in only $73 \%$ of their patients.

In the absence of the major diagnostic criterion, compact mast cell infiltrates, KIT D816V served as the critical third minor diagnostic criterion in $20 \%$ of our patients (12/59), thus allowing a final diagnosis of systemic mastocytosis. ${ }^{1}$ The lack of detectable compact mast cell infiltrates in the bone marrow poses a diagnostic challenge in patients with suspected systemic mastocytosis. It has been reported in up to $25 \%$ of patients by others and was noted in $29 \%$ of patients $(17 / 59)$ in our study. ${ }^{13}$ Arguing in favor of a sampling error, Butterfield and $\mathrm{Li}$ demonstrated that the diagnosis of 'mastocytosis' would have been missed in 17\% (4/23) of their patients who underwent bilateral bone marrow biopsy, if only a single biopsy specimen had been obtained. ${ }^{14}$

The patients without detectable compact mast cell infiltrates are usually characterized by a lower mast cell burden, which, in turn, results in only a slight and often sub-diagnostic elevation of the serum tryptase level. ${ }^{14}$ Sixty-five percent (11/17) of our compact mast cell infiltrate-negative patients had serum tryptase levels below the threshold of $20 \mathrm{ng} / \mathrm{ml}$, compared with only $29 \%(12 / 42)$ in the compact mast cell infiltrate-positive group. Thus, both compact mast cell infiltrates and serum tryptase were diagnostically inconclusive in about $19 \%$ $(11 / 59)$ of our patients with suspected systemic mastocytosis. Positive results for all three remaining minor criteria, that is, atypical mast cell morphology, expression of CD25, and KIT D816V, are in these cases essential to establish a final diagnosis of systemic mastocytosis.

Detection of KIT D816V is a technical challenge in patients without detectable compact mast cell infiltrates. In these patients, KIT mutations are almost always restricted to neoplastic mast cells and their precursors. ${ }^{15,16}$ The very high background of KIT wild-type cells warrants a very high diagnostic sensitivity for detection of KIT D816V. It may best be achieved by preferential amplification of the mutated alleles. ${ }^{17-19}$ Nevertheless, in a significant number of cases, KIT mutational analysis will need to be performed on mast cells enriched by either flow cytometric sorting of bone marrow aspirate cells or laser microdissection of mast cells from tissue sections. ${ }^{6,20,21}$ In our study, the diagnostic sensitivity for KIT D816V was thereby increased from $83 \%(48 / 58)$ to $100 \%(58 / 58)$ and allowed the diagnosis of systemic mastocytosis to be established in four additional patients (4/58, 6.9\%). As expected for the less sensitive techniques investigating DNA from complete tissue sections, the diagnostic sensitivity for KIT D816V in the bone marrow correlated significantly with the mast cell burden and the presence of compact mast cell infiltrates $(P<0.001)$. The mast cell burden, in turn, correlated significantly with the serum tryptase level $(P=0.002) .{ }^{4}$

KIT D816V is known to be expressed in abnormal skin mast cells in urticaria pigmentosa. ${ }^{22}$ We failed to detect KIT D816V in microdissected skin mast cells in about $25 \%$ of cases despite the fact that it was detectable in bone marrow mast cells. Consistent with the decreased detection rate of KIT mutations in the skin is the observation that only a small subset of skin mast cells expressed CD25 as another indicator of the neoplastic nature of these cells, ${ }^{9,10,23}$ while it was found to be strongly expressed by the majority of bone marrow mast cells (in the present study) and mucosal mast cells in patients with intestinal involvement of systemic mastocytosis. ${ }^{24}$ These findings may be caused by, for example, expression of different sets of adhesion molecules between normal and neoplastic mast cells, or by expression of different homing receptors for (neoplastic) mast cells in the skin and extracutaneous tissues. ${ }^{25,26}$ Consequently, the finding of KIT wild type in the skin of adult patients with mastocytosis in the skin neither 
correlated with mast cell density in the skin or bone marrow nor with skin lesion intensity or tryptase levels. Most importantly, it did not exclude systemic mastocytosis.

The clinical implications of the diagnosis of systemic mastocytosis are substantial. Even though patients with indolent systemic mastocytosis have an almost normal life expectancy, significant danger may arise from anaphylactic reactions..$^{27-30}$ In a recent study, Brockow et al. ${ }^{30}$ found a cumulative incidence of anaphylaxis of up to $49 \%$ in adults with systemic mastocytosis leading to the recommendation to carry an emergency medication set, including epinephrine. Severe and even fatal intra- and postoperative complications also pose an important threat. ${ }^{31-33}$ In addition, patients with systemic mastocytosis should be monitored closely for osteopenia and osteoporosis, which may lead to pathological fractures. ${ }^{34}$

In summary, we demonstrate, for the first time, that almost all patients with adult-onset mastocytosis in the skin, usually presenting as urticaria pigmentosa, suffer from systemic mastocytosis as defined by the diagnostic WHO criteria. Even in cases lacking sufficient diagnostic criteria for systemic mastocytosis, clonality of bone marrow mast cells could be demonstrated by the detection of KIT D816V in these cells, suggesting the presence of a generalized clonal mast cell disease. According to a recent consensus proposal, a monoclonal mast cell activation syndrome may be diagnosed in these patients, provided that they suffer from mast cell mediator-related symptoms. ${ }^{35}$ Our data challenge the concept of pure adult-onset cutaneous mastocytosis and demonstrate that a molecular finding of KIT wild type in skin biopsies does not exclude systemic mastocytosis. Therefore, the typical skin lesions of cutaneous mastocytosis in adult patients may indeed represent a finding in favor of the diagnosis 'systemic mastocytosis' and could thus possibly be considered as an additional (minor) diagnostic criterion for systemic mastocytosis in a future consensus classification. However, as histological features of mastocytosis in the skin are still ill-defined, in contrast to the diagnostic criteria in extracutaneous tissues, bone marrow histology should be performed in every case of mastocytosis in the skin to verify the diagnosis of systemic mastocytosis and to allow its subtyping.

\section{Acknowledgements}

We thank Elena Born and Sema Colak for excellent technical assistance. We also thank Dr. Margaret Ruck for language editing the manuscript. SB and MJF contributed equally to this manuscript. The study was partly supported by grants from the K. L. Weiland'sche Stiftung, LMU, Curt-Bohnewands-Fonds, and Georg und Traud Gravenhorst Stiftung.

\section{Disclosure/conflict of interest}

The authors declare no conflict of interest.

\section{References}

1 Horny HP, Metcalfe DD, Bennett JM, et al. Mastocytosis, In: Swerdlow SH, Campo E, Harris NL, et al. (eds) WHO Classification of Tumours of Haematopoietic and Lymphoid Tissues, 4th edn. International Agency for Research and Development (IARC): Lyon, France; 2008, pp 54-63.

2 Valent P, Akin C, Escribano L, et al. Standards and standardization in mastocytosis: consensus statements on diagnostics, treatment recommendations and response criteria. Eur J Clin Invest 2007;37:435-453.

3 Escribano L, Alvarez-Twose I, Sanchez-Munoz L, et al. Prognosis in adult indolent systemic mastocytosis: a long-term study of the Spanish Network on Mastocytosis in a series of 145 patients. J Allergy Clin Immunol 2009;124:514-521.

4 Brockow K, Akin C, Huber M, et al. Assessment of the extent of cutaneous involvement in children and adults with mastocytosis: relationship to symptomatology, tryptase levels, and bone marrow pathology. J Am Acad Dermatol 2003;48:508-516.

5 Sperr WR, Escribano L, Jordan JH, et al. Morphologic properties of neoplastic mast cells: delineation of stages of maturation and implication for cytological grading of mastocytosis. Leuk Res 2001;25: 529-536.

6 Sotlar K, Colak S, Bache A, et al. Variable presence of KITD816V in clonal haematological non-mast cell lineage diseases associated with systemic mastocytosis (SM-AHNMD). J Pathol 2010;220:586-595.

7 Sotlar K, Escribano L, Landt O, et al. One-step detection of c-kit point mutations using peptide nucleic acid-mediated polymerase chain reaction clamping and hybridization probes. Am J Pathol 2003;162:737-746.

8 Sotlar K, Bache A, Stellmacher F, et al. Systemic mastocytosis associated with chronic idiopathic myelofibrosis: a distinct subtype of systemic mastocytosis associated with a [corrected] clonal hematological non-mast [corrected] cell lineage disorder carrying the activating point mutations KITD816V and JAK2V617F. J Mol Diagn 2008;10:58-66.

9 Lanternier F, Cohen-Akenine A, Palmerini F, et al. Phenotypic and genotypic characteristics of mastocytosis according to the age of onset. PLoS One 2008; 3:e1906.

10 Hollmann TJ, Brenn T, Hornick JL. CD25 expression on cutaneous mast cells from adult patients presenting with urticaria pigmentosa is predictive of systemic mastocytosis. Am J Surg Pathol 2008;32:139-145.

11 Tebbe B, Stavropoulos PG, Krasagakis K, et al. Cutaneous mastocytosis in adults. evaluation of 14 patients with respect to systemic disease manifestations. Dermatology 1998;197:101-108.

12 Travis WD, Li CY, Su WP. Adult-onset urticaria pigmentosa and systemic mast cell disease. Am J Clin Pathol 1985;84:710-714.

13 Sanchez-Munoz L, Alvarez-Twose I, Garcia-Montero AC, et al. Evaluation of the WHO criteria for the classification of patients with mastocytosis. Mod Pathol 2011;24: 1157-1168. 
14 Butterfield JH, Li CY. Bone marrow biopsies for the diagnosis of systemic mastocytosis: is one biopsy sufficient? Am J Clin Pathol 2004;121:264-267.

15 Hartmann K, Wardelmann E, Ma Y, et al. Novel germline mutation of KIT associated with familial gastrointestinal stromal tumors and mastocytosis. Gastroenterology 2005;129:1042-1046.

16 Orfao A, Garcia-Montero AC, Sanchez L, et al. Recent advances in the understanding of mastocytosis: the role of KIT mutations. Br J Haematol 2007;138:12-30.

17 Kahler C, Didlaukat S, Feller AC, et al. Sensitive and reliable detection of Kit point mutation Asp 816 to Val in pathological material. Diagn Pathol 2007;2:37.

18 Schumacher JA, Elenitoba-Johnson KS, Lim MS. Detection of the c-kit D816V mutation in systemic mastocytosis by allele-specific PCR. J Clin Pathol 2008;61:109-114.

19 Tan A, Westerman D, McArthur GA, et al. Sensitive detection of KIT D816V in patients with mastocytosis. Clin Chem 2006;52:2250-2257.

20 Teodosio C, Garcia-Montero AC, Jara-Acevedo M, et al. Mast cells from different molecular and prognostic subtypes of systemic mastocytosis display distinct immunophenotypes. J Allergy Clin Immunol 2010;125: 726 e711-726 e714.

21 Garcia-Montero AC, Jara-Acevedo M, Teodosio C, et al. KIT mutation in mast cells and other bone marrow hematopoietic cell lineages in systemic mast cell disorders: a prospective study of the Spanish Network on Mastocytosis (REMA) in a series of 113 patients. Blood 2006;108:2366-2372.

22 Longley BJ Jr., Metcalfe DD, Tharp M, et al. Activating and dominant inactivating c-KIT catalytic domain mutations in distinct clinical forms of human mastocytosis. Proc Natl Acad Sci USA 1999;96:1609-1614.

23 Sundram UN, Natkunam Y. Mast cell tryptase and microphthalmia transcription factor effectively discriminate cutaneous mast cell disease from myeloid leukemia cutis. J Cutan Pathol 2007;34:289-295.

24 Hahn HP, Hornick JL. Immunoreactivity for CD25 in gastrointestinal mucosal mast cells is specific for systemic mastocytosis. Am J Surg Pathol 2007;31: 1669-1676.

25 Hallgren J, Gurish MF. Pathways of murine mast cell development and trafficking: tracking the roots and routes of the mast cell. Immunol Rev 2007;217:8-18.

26 Kim MS, Radinger M, Gilfillan AM. The multiple roles of phosphoinositide 3-kinase in mast cell biology. Trends Immunol 2008;29:493-501.

27 Ludolph-Hauser D, Rueff F, Fries C, et al. Constitutively raised serum concentrations of mast-cell tryptase and severe anaphylactic reactions to Hymenoptera stings. Lancet 2001;357:361-362.

$28 \mathrm{Lim}$ KH, Tefferi A, Lasho TL, et al. Systemic mastocytosis in 342 consecutive adults: survival studies and prognostic factors. Blood 2009;113:5727-5736.

29 Niedoszytko M, de Monchy J, van Doormaal JJ, et al. Mastocytosis and insect venom allergy: diagnosis, safety and efficacy of venom immunotherapy. Allergy 2009;64:1237-1245.

30 Brockow K, Jofer C, Behrendt H, et al. Anaphylaxis in patients with mastocytosis: a study on history, clinical features and risk factors in 120 patients. Allergy 2008;63:226-232.

31 Chaar CI, Bell RL, Duffy TP, et al. Guidelines for safe surgery in patients with systemic mastocytosis. Am Surg 2009;75:74-80.

32 Damodar S, John CN, Gopalakrishnan G, et al. Mast cell disease: surgical and anesthetic implications. J Pediatr Hematol Oncol 2006;28:446-449.

33 Yunaev M, Hughes TM, Abdul-Razak M. Systemic mastocytosis and surgery a potential disaster. ANZ J Surg 2010;80:860-861.

34 van der Veer E, van der Goot W, de Monchy JG, et al. High prevalence of fractures and osteoporosis in patients with indolent systemic mastocytosis. Allergy 2012;67:431-438.

35 Valent P, Akin C, Arock M, et al. Definitions, criteria and global classification of mast cell disorders with special reference to mast cell activation syndromes: a consensus proposal. Int Arch Allergy Immunol 2012;157:215-225. 